\title{
On the polarisation of the Red Rectangle optical emission bands ${ }^{\star}$ (Research Note)
}

\author{
N. L. J. Cox ${ }^{1}$, B. H. Foing ${ }^{2}$, J. Cami ${ }^{3,5}$, and P. J. Sarre ${ }^{4}$ \\ 1 Instituut voor Sterrenkunde, K.U. Leuven, Celestijnenlaan 200D, 3001 Leuven, Belgium \\ e-mail: nick@ster.kuleuven. be \\ 2 ESA Research and Scientific Support Department, PO Box 299, 2200-AG Noordwijk, The Netherlands \\ 3 Department of Physics \& Astronomy, University of Western Ontario, London, ON N6A 3K7, Canada \\ ${ }^{4}$ School of Chemistry, The University of Nottingham, University Park, Nottingham, NG7 2RD, UK \\ 5 SETI Institute, 189 Bernardo Ave., Suite 100, Mountain View, CA 94043, USA
}

Received 9 March 2011 / Accepted 20 May 2011

\begin{abstract}
Context. The origin of the narrow optical emission bands seen towards the Red Rectangle is not yet understood. We investigate the proposal that these are caused by luminescence of large carbonaceous molecules.

Aims. We aim to measure the polarisation of the optical narrow Red Rectangle bands (RRBs). Polarised signals of several percent could be expected from certain asymmetric molecular rotators.

Methods. The ESPaDOnS échelle spectrograph mounted at the CFHT was used to obtain high-resolution optical spectropolarimetric data of the Red Rectangle nebular emission.

Results. The RRBs at 5800, 5850, and $6615 \AA$ are detected in spectra of the nebular emission 7" and 13" north-east from the central star. The $5826 \AA$ and $6635 \AA$ RRB are detected only at the position nearest to the central star. For both positions the Stokes $Q$ and $U$ spectra show no unambiguous polarisation signal in any of the RRBs. We derive an upper limit of $0.02 \%$ line polarisation for these RRBs. A tentative feature with peak polarisation of $0.05 \%$ is seen for the $5800 \AA$ RRB at 7 " offset. However, the null spectra suggest that this may be an instrumental artefact.

Conclusions. The lack of a clear polarisation signal for the five detected RRBs implies that if the emission is caused by luminescence of complex organics, these gas-phase molecular carriers are likely to have a high degree of symmetry because they do not exhibit a Q-branch in their rotational profile, although this may be modified by statistical effects.
\end{abstract}

Key words. astrochemistry - polarization - circumstellar matter - stars: individual: Red Rectangle - line: profiles

\section{Introduction}

The Red Rectangle (RR) is a unique bi-conical reflection nebula (Cohen et al. 2004). At its centre lies a close binary system of which the primary (HD 44179) is a luminous evolved star. The system is visually obscured by a thick and massive circumbinary dusty disk that is seen nearly edge-on (Waters et al. 1998). Interactions between the stellar wind and disk result in the X-shaped nebula. The central star is of 9th visual magnitude, while the nebula has a surface $V$ brightness of about 19 mag per square arcsecond (e.g. Schmidt \& Witt 1991).

The infrared (IR) spectrum of the RR nebula shows strong infrared emission bands across the near- and mid-infrared spectral region (Merrill 1977; Russell et al. 1977, 1978; Geballe et al. 1989; Waelkens et al. 1996; Hony et al. 2001; Peeters et al. 2002; Song et al. 2007). These are generally accepted to be caused by vibrational transitions of PAHs (see review by Tielens 2008). Observations show that the aromatic emission in the RR

* Based on observations obtained at the Canada-France-Hawaii Telescope (CFHT) which is operated by the National Research Council of Canada, the Institut National des Sciences de l'Univers of the Centre National de la Recherche Scientique of France, and the University of Hawaii. originates from the extended nebular emission of the RR, similar to the RRBs (Sloan et al. 1993; Waters et al. 1998).

At optical wavelengths, the RR was the first object in which extended red emission (ERE) was detected (Cohen et al. 1975; Greenstein \& Oke 1977; Schmidt et al. 1980; Schmidt \& Witt 1991). The carriers of this broad emission in the red part of the spectrum have not been identified, but it is believed that ERE is caused by photoluminescence from materials such as PAHs, quenched carbonaceous composites, hydrogenated amorphous carbon, carbon clusters, nanodiamonds, etcetera (d'Hendecourt et al. 1986; Witt \& Boroson 1990; Furton \& Witt 1990; Witt et al. 2006). Note that the ERE has also been detected in the diffuse ISM (Gordon et al. 1998) and in compact $\mathrm{H}_{\text {II }}$ regions (Darbon et al. 2000). In addition, blue luminescence (BL) between about 3900 and $4100 \AA$ has also been detected towards both the RR and several reflection nebulae, and has been attributed specifically to fluorescence of (neutral) PAHs containing 14 to 18 carbon atoms (Vijh et al. 2004, 2005). Recent laboratory experiments suggest that BL is caused by small gas-phase PAHs, while ERE is caused by larger PAHs in grains (Wada et al. 2009).

Superposed on the ERE in the Red Rectangle are a plethora of narrow unidentified emission features (Schmidt et al. 1980; Warren-Smith et al. 1981), whose widths, profile shapes and peak positions change as a function of the distance from the 
central star (Schmidt \& Witt 1991; Scarrott et al. 1992; Sarre et al. 1995; Glinski \& Anderson 2002; Van Winckel et al. 2002) and are called the Red Rectangle bands (RRBs). The ERE and the RRBs are most pronounced along the bi-conical interfaces although their spatial distributions are not identical (Schmidt $\&$ Witt 1991). As one moves away from the central star along the bi-conical interface, the wavelengths of the RRBs shift bluewards (Glinski \& Anderson 2002; Van Winckel et al. 2002).

Schmidt et al. (1980) suggested that the sharp emission features (e.g. near 5800 and $6615 \AA$ ) may arise in gaseous molecules, whereas the diffuse underlying (ERE) component would correspond to the same transition (but somewhat perturbed) in molecules attached to grains. The RRBs are nebular emission rather than scattered starlight. Sharp et al. (2006) illustrate that the RRBs can be reproduced, for example, by emission from the (vibrational) "sequence structure" of large fluorescent PAH molecules, where overlapping vibrational contours at different vibrational temperatures cause the observed variations across the nebula. To the best of our knowledge, the polarisation properties of the bands have not been investigated before. A recent assesment of the Red Rectangle band problem is given in Glinski et al. (2009), who argue that some aspects of the RRB behaviour may be be caused by 1) self-absorption (cold material in front of hotter material); 2) velocity dispersion within the nebula (broadening of intrinsic line profile); or 3) overlapping of bands from different weak emitters.

A key question for the Red Rectangle emission bands (and diffuse interstellar absorption bands) is whether the spectral line carriers are free gas-phase molecules or if they are directly associated with dust grains. This latter scenario could hold if the carriers were within or on the surface of the grains. Interstellar grains cause polarisation of starlight, and consequently polarimetric measurements on the Red Rectangle bands allow this to be probed.

In addition to the intrinsic interest in understanding and assignment of the RRBs, some of the prominent RRBs at 5800, 6380 , and $6615 \AA$ are very close in wavelength (where the differences decrease with increasing distance from the central star) to a set of strong narrow, but diffuse, interstellar absorption lines at 5797, 6379 and $6614 \AA$. This coincidence has led to the hypothesis that there may be a direct link between the RRBs and some of the ubiquitous diffuse interstellar bands (Fossey 1991; Sarre 1991; Scarrott et al. 1992; Sarre et al. 1995). Diffuse band carriers are also believed to be large, stable carbonaceous interstellar gas-phase molecules (Sarre 2006), and any connection between DIBs and RRBs could therefore provide further insight in either carrier. For now both their identity and connection to the RRB are open questions.

Broad-band spectropolarimetry $11^{\prime \prime}$ south of the central star revealed a reduction in the degree of polarisation (from $6 \%$ in the blue down to $2 \%$ at the ERE wavelength) but constant position angle $\left(\sim 78^{\circ}\right)$. This suggests that the emission originates within the nebula itself and dilutes the polarised scattered light from the central star (Schmidt et al. 1980; Gledhill et al. 2009). Substantial polarisation $(\sim 20 \%)$ is observed along the conic interface just outside the bicone, while values of 5-10\% are common in the nebula itself, although the ERE component is likely unpolarised (Perkins et al. 1981). Reese \& Sitko (1996) measured a linear continuum polarisation level of $2.2 \pm 1.1 \%$ towards the central star.

From theoretical work and laboratory experiments it has been found that fluorescent emission of large molecules can exhibit linear polarisation changes along a set of vibronic lines (Tolkachev \& Pliska 1986; Tolkachev \& Polubisok 1995, 1998; Tolkachev 1998a,b, 2002; Borisevich et al. 2002, 2006; Tolkachev \& Blokhin 2009). The overall degree of polarisation as well as the variation across the rotational contour strongly depend on the molecular symmetry (see also Nathanson \& McClelland 1986): extreme prolate or oblate molecules could display polarisations of up to $20 \%$. Very symmetrical species on the other hand (or indeed dust particles) are not expected to exhibit much polarisation across the lines. Sironi \& Draine (2009) showed that the infrared emission of PAHs could become polarised, albeit at low levels of $<0.1 \%$, upon anisotropic illumination by UV photons. This offers tantalizing prospects for constraining the geometry (shape) of the RRB carriers by measuring the linear line polarisation in space for the first time.

This paper presents a spectropolarimetric study of the nebulosity of the Red Rectangle. After a presentation of the observations we discuss the implications of our results for the properties of the carriers of the optical narrow emission bands (RRBs) and, by implication, possibly also those of the DIB and UIB carriers.

\section{Spectropolarimetric observations}

We obtained new spectropolarimetry data with ESPaDOnS at the Canadian-French-Hawaian Telescope (CFHT). The data were taken in service mode during several nights in January and February 2010. ESPaDOnS is a high-resolution high-efficiency two-fiber echelle spectrograph with polarising capabilities. The resolution for the spectropolarimeter is about 64000 , covering a wide spectral range from 3700 to $10480 \AA$ with only a few small gaps in the near-infrared. The fiber (1.6" diameter) was placed first $4.5^{\prime \prime}$ north, $5^{\prime \prime}$ east and secondly $9^{\prime \prime}$ north, $9^{\prime \prime}$ east along the bi-conical interface, relative to central star. In the following we refer to these two nebular positions as $\sim 7^{\prime \prime}$ and $\sim 13^{\prime \prime}$ offset from the central star along the biconical interface in the north-east direction. For each Stokes parameter the observing sequence consists of four spectra obtained at different orientations of the prism. For both linear Stokes parameters (i.e. $Q$ and $U$ ) we obtained three sequences of $4 \times 1000 \mathrm{~s}$ at the 7 " position (i.e. $12000 \mathrm{~s}$ total exposure) and four sequences of $4 \times 1200 \mathrm{~s}$ at the $13^{\prime \prime}$ position (19200 s total). In addition, for reference, we obtained Stokes $Q$ and $U$ spectra of the central star, HD 44179, in a similar fashion (5040 s total).

The observations were automatically reduced with Upena, which is the CFHT's reduction pipeline for ESPaDOnS. The Upena data reduction system uses Libre-ESpRIT, which is a purpose built (proprietary) data reduction software tool (Donati et al. 1997). Owing to the low flux levels of the extended emission, the automatic continuum normalisation did not perform well and was therefore not used. Absolute flux calibration was not performed. The relative spectral response was obtained via normal flat-fielding. At the selected positions the nebula has a low surface brightness and only a weak spectral signal is received. The heliocentric velocity correction and the radial velocity correction from telluric lines are automatically applied. Next, the individual spectra were rebinned (to a constant wavelength grid with a pixel size of $0.03 \AA$ at $5800 \AA$ and to $0.04 \AA$ at $6600 \AA$ ) and cosmic spikes removed. The spectra were then averaged with weights taken from the uncertainties given for the total intensity. The averaged spectra at 7" reveal the RRBs peaking at 5799, 5826, 5854, 6615, and $6635 \AA$. Additional weaker RRBs have been revealed in previous studies by, e.g. Van Winckel et al. (2002), but these do not show up clearly in our spectra. 
We note that there are small differences in the strengths and shapes of the nebular emission lines between individual spectra. These are most likely casued by unavoidable (positional) differences between different exposure sequences, in particular those taken on different nights. The four spectra taken within a single sequence do not suffer from shifts because the hole was kept fixed in position with respect to the central star using Cassegrain tracking. The total error-weighted average spectra are continuum-subtracted by measuring the continuum levels between 5780-5790 ̊ and 6605-6610 A, respectively.

The Stokes $I, Q$, and $U$ spectra covering the optical emission bands at $5800 \AA$ (including also the RRB at $5825 \AA$ ) and $6615 \AA$ are shown in Figs. 1 and 2 for both nebular positions, respectively. In addition, the check spectra N2(Q) and N2(U) are shown to identify possible spurious polarisation signals. For clarity the N1 spectra are not show in the above figures because their appearance is similar to the N2 spectra. The null spectra (N1 and N2) are a diagnostic of possible instrumental artefacts linked to the intensity or Stokes parameter, respectively. Preliminary studies show that for intense emission lines in Stokes $I$ an associated structure in the null spectra does not necessarily discredit the detection of signatures in the Stokes spectra (Fabas 2008). The central star spectra are displayed at the top in Fig. 1. Features are tentatively identified at the position of the 5780, 5797, and $6614 \AA$ DIBs. We identify the three strong features at 5793,5801 , and $6611.5 \AA$ with photospheric $\mathrm{C}_{\mathrm{I}}$, which is known to be present at suprasolar abundances in HD 44179 (Waelkens et al. 1992).

\section{Discussion}

\section{$R R B$ total intensity profiles}

The two RRBs at $5800 \AA$ and $6615 \AA$ are clearly detected at both positions in the nebula (Figs. 1 and 2). The spectra taken at the larger offset show weaker RRBs. The intrinsically weaker and broader RRBs at 5826 and $6635 \AA$ are only seen at the position nearest to the central star. At 13" a narrow emission component is detected around $6635 \AA$.

The emission velocity profiles for the 5800, 5850, and $6615 \AA$ RRBs are very similar for each offset position as is illustrated in Fig. 3. Their peak flux ratios are 1:2.5:1.75, respectively. The FWHM of these RRBs is about $13 \mathrm{~cm}^{-1}$ $\left(\sim 250 \mathrm{~km} \mathrm{~s}^{-1}\right)$ at $7^{\prime \prime}$ offset. Closer to the star these RRBs have a pronounced red wing that is not present at 13" from the central source. At $7^{\prime \prime}$ the profiles show a steep blue edge as well as a broad red-wing, while farther away from the star at $13^{\prime \prime}$ the wing has disappeared and a weak narrow component (shifted bluewards) remains. The red wing of the $5800 \AA$ feature appears slightly more enhanced with respect to the two other RRBs. This behaviour is consistent with the blueshift in the RRB feature and decrease in the $F W H M$ for increasing offsets from the central star as discussed by, e.g. Glinski \& Anderson (2002) and Van Winckel et al. (2002).

The appearance of a red wing is consistent with the common observation of vibrational sequence bands in electronic spectra where the vibrational frequencies in the emitting state are slightly lower than in the ground state. With an increase in offset, the populations of vibrationally excited levels in the ground electronic state will decline, resulting in reduced optical excitation to vibrationally excited levels of the excited electronic state. Consequently, a reduction in sequence band emission intensity with offset would be anticipated and is consistent with narrowing
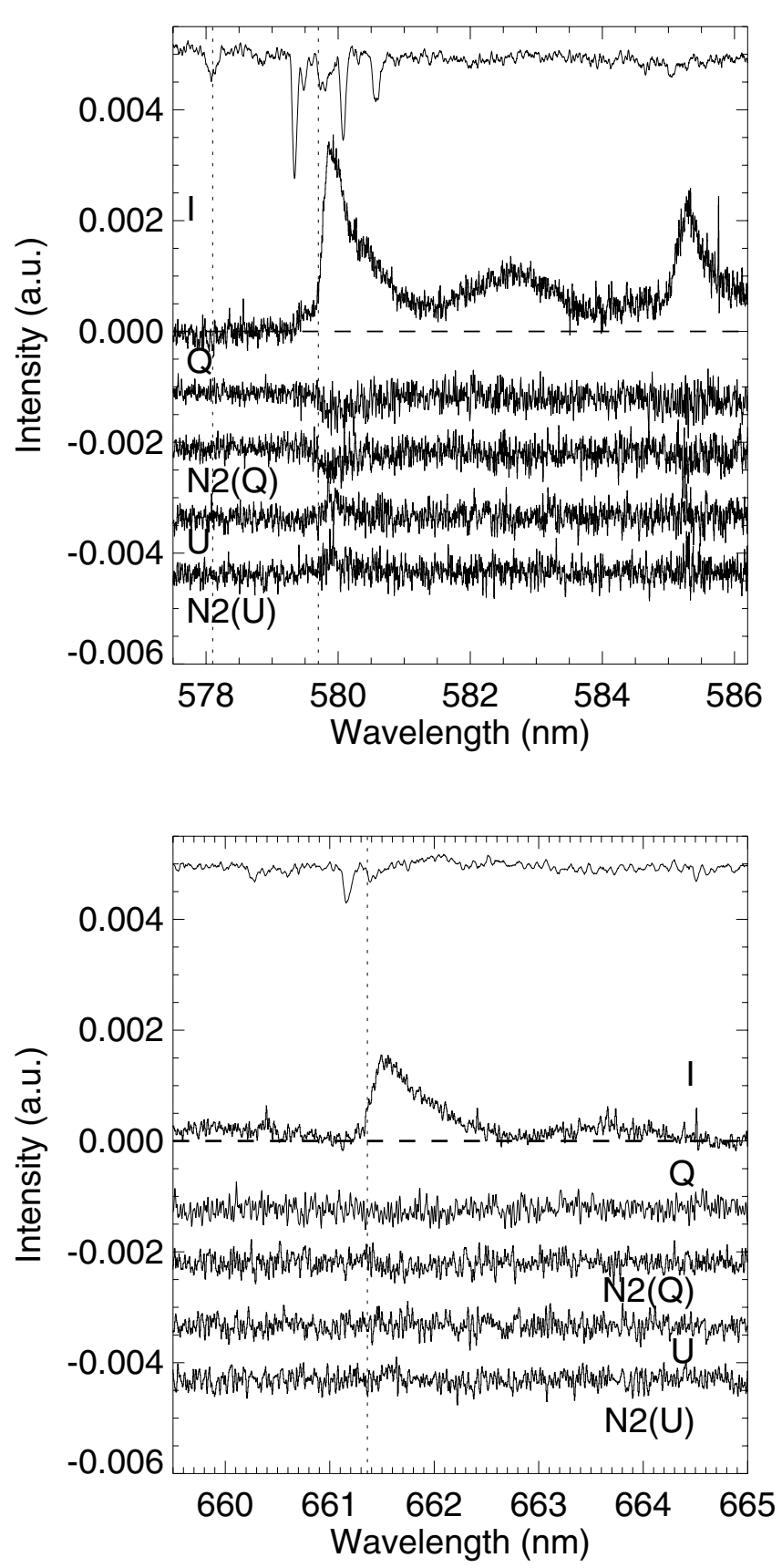

Fig. 1. Stokes $I, Q$, and $U$ spectra of the 5800 and $5826 \AA$ (top), and $6615 \AA$ (bottom) RRBs at an offset of 7". For the displayed spectra a three-pixel smoothing function was applied. The spectrum (continuum normalised and arbitrarily scaled) at the top is that of the central star.

of the profile. The blueward edge is relatively unaffected by a reduction in the internal vibrational temperature with offset.

In contrast to the 5800, 5850, and 6615 bands, the profiles of the 5825 and $6635 \AA$ RRBs (at $7^{\prime \prime}$ ) have symmetrical shapes with FWHM of $\sim 24 \mathrm{~cm}^{-1}$ (Fig. 4). These two bands appear to be satellites of the 5800 and 6615 RRBs, respectively, and within this interpretation could arise from transitions from the excited vibronic (vibrationless) levels to one or more vibrationally excited levels in the ground electronic states of the carriers. The separations between the 5800 and $5825 \AA\left(74 \mathrm{~cm}^{-1}\right)$ and 6615 and $6635 \AA\left(46 \mathrm{~cm}^{-1}\right)$ bands would then be vibrational intervals 

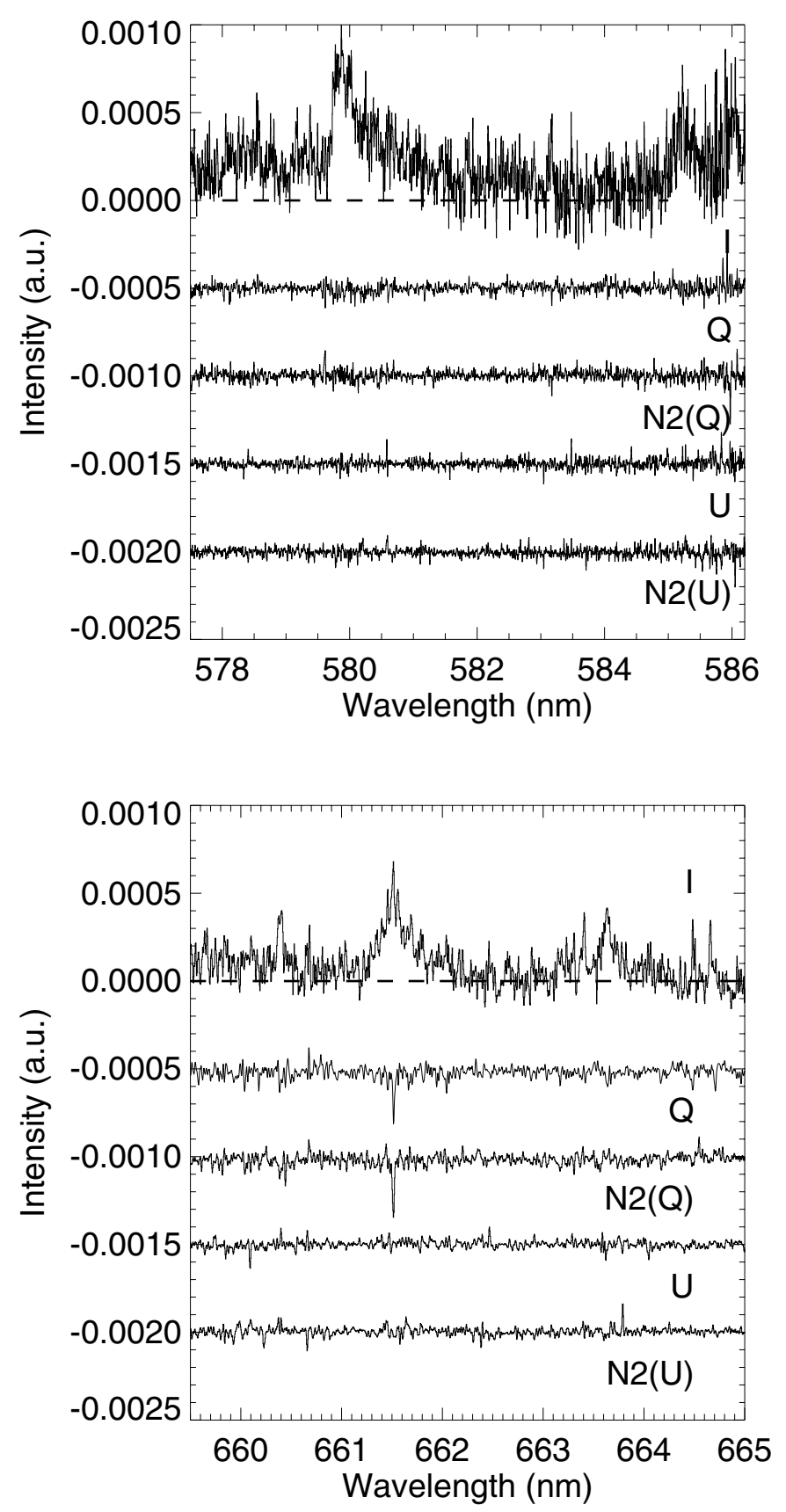

Fig. 2. Stokes $I, Q$, and $U$ spectra of the $5800 \AA$ (top) and $6616 \AA$ (bottom) RRBs at an offset of $13^{\prime \prime}$. For the displayed spectra a three-pixel smoothing function was applied. In the average intensity spectrum a narrow feature appears at the location of the broad $6635 \AA$ RRB. The very sharp peaks are probably instrumental artefacts.

in the respective ground electronic states of the carriers. The band intensities of the two satellite bands are too low in these data to determine their behaviour as a function of offset. The fact that they are symmetrical may be significant, although it could result simply from the overlap of multiple bands.

\section{RRB polarisation features}

Inspection of the averaged polarisation spectra at 7" shows a small polarisation feature in the $5800 \AA$ RRB for both Stokes $Q$
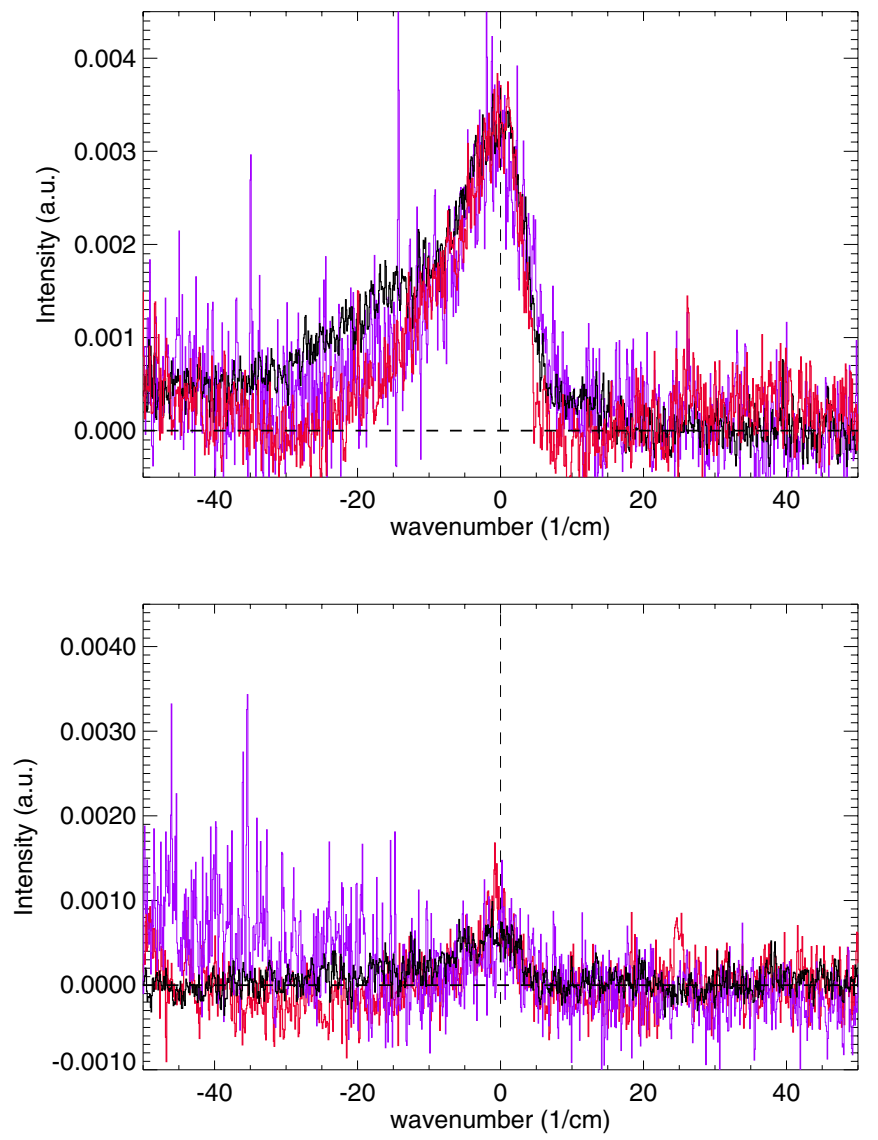

Fig. 3. Intensity-wavenumber profiles for the 5800 (black), 5850 (purple), and $6615 \AA$ (red) RRBs compared for the 7" (top) and 13" (bottom) offset positions. The continuum-subtracted profiles were shifted in wavenumber to optimise the comparison. At 7" the zero wavenumbers for the three RRBs correspond to 5798.9, 5852.7, and 6615.4 $\AA$. And at $13^{\prime \prime}$ the zero wavenumbers correspond to 5798.3, 5852.3, and $6614.8 \AA$, respectively. The 5850 and $6615 \AA$ RRB profiles (at both offset positions) were scaled by factors of 1.75 and 2.5 , respectively, to match the peak intensity of the $5800 \AA$ RRB. In this figure the displayed spectra were not smoothed. Both panels are shown in identical wavenumber and intensity ranges. Note also the "inversed" profiles with respect to those plotted as a function of wavelength.

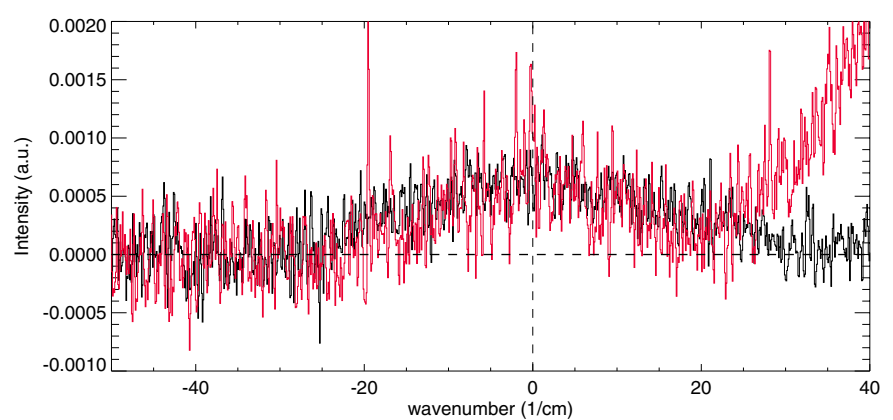

Fig. 4. Intensity-wavenumber profiles for the 5825 and $6635 \AA$ RRBs are compared to each other for the 7" offset position. Each profile was shifted in wavenumber to put the peak at $0 \mathrm{~cm}^{-1}$. The peak wavelengths correspond to 5826.5 and $6636.5 \AA$, respectively. The $6635 \AA$ RRB profile was scaled by a factor of 2.5 to match the peak intensity of the $5825 \AA$ RRB. In this figure the displayed spectra were not smoothed. 
and $U$ spectra. However, because these features also appear in the check N1 and N2 spectra, they cannot be confirmed to be non-artificial (i.e. caused by instrumental effects and not intrinsic to the source). Preliminary investigations of ESPaDOnS data have shown that intense emission in Stokes $I$ could be associated with a structure in the $\mathrm{N}$ channels, without putting the reliability of the detected signatures in Stokes parameter into doubt (Fabas 2008). This needs to be investigated more closely. If these features were real polarisation features intrinsic to the emission profile, we note that the peak polarisation level of this feature would be $\sim 0.05 \%$, with a $1-\sigma$ noise on the polarisation of about $\sim 0.02 \%$. None of the other RRBs at 5825, 5850, and $6615 \AA$ show any sign of polarisation features (Figs. 1 and 2). The absence of line polarisation in the RRBs suggests that they are not directly related to dust grains. The expected levels of line polarisation would be similar to those of the continuum $(\sim 2 \%)$. For fluorescent emission from asymmetric molecules (i.e. with a rotational Q-branch) excess line polarisation, up to $20 \%$, in the Q-branch with respect to the P- and R-branches is predicted (Tolkachev 1998b). We conclude that the RRBs are likely not caused by grains or molecules with strong Q-branches (such as PAHs with side-groups; amino-acids, etc.). Possibly the RRB carriers are more symmetric (thus lacking a Q-branch) because of photo-destruction of side-groups in the harsh circumstellar UV radiation environment of the Red Rectangle. Nonetheless, the results are also consistent with the carriers being large gasphase molecules (even those with a high degree of symmetry) because statistical effects then play a more significant role in reducing the level of fluorescence polarisation.

\section{Conclusion}

The spectropolarimetric observations presented here give new insight in the properties of the Red Rectangle bands (RRBs). The high-resolution spectra show that (as suspected from lower resolution spectra) the profiles of the 5800,5850 , and $6615 \AA$ RRBs are very similar. Although the profiles change with distance from the central source, this similarity holds for both positions. These high-resolution spectra reveal no profile substructure except for the appearance of a red wing, as observed before, at the smallest offset position.

The main result is that the Red Rectangle bands at 5800, $5825,5850,6615$ and $6635 \AA$ are not polarised down to levels of $0.02 \%$. This implies that

- The carriers of the RRBs are not directly attached to the dust grains because the latter show significant continuum polarisation.

- The lack of strong narrow polarisation features further suggests that the Q-branch emission does not make up part of the observed profile.

- The carriers are large molecules because statistical effects then play a more significant role in reducing the level of fluorescence polarisation. On the other hand, these transitions could be caused by a non-vibronic origin band electronic transition, even for a symmetric molecule.

Nonetheless, it is known that P- and R-branches giving rise to fluorescent emission can show line polarisation at very low levels. Thus, additional higher signal-to-noise spectropolarimetry at different positions in the extended emission is needed to establish the reality of the tentative polarisation feature related to the $5800 \AA \mathrm{RRB}$ and to probe in more detail the polarisation of all known RRBs because they do not necessarily share identical carriers.

Acknowledgements. We thank the QSO staff - in particular Nadine Manset - at the CFHT for advice on the preparation for the observing run and on technical aspects of the processing of ESPaDOnS data. We thank the referee for helpful comments.

\section{References}

Borisevich, N. A., Derzhitskii, S. L., Povedailo, V. A., \& Tolkachev, V. A. 2002, J. Appl. Spec., 69, 571

Borisevich, N. A., Povedailo, V. A., Tolkachev, V. A., \& Yakovlev, D. L. 2006, Physics - Doklady, 51, 17

Cohen, M., Anderson, C. M., Cowley, A., et al. 1975, ApJ, 196, 179

Cohen, M., Van Winckel, H., Bond, H. E., \& Gull, T. R. 2004, AJ, 127, 2362

Darbon, S., Zavagno, A., Perrin, J., et al. 2000, A\&A, 364, 723

d'Hendecourt, L. B., Léger, A., Olofsson, G., \& Schmidt, W. 1986, A\&A, 170, 91

Donati, J.-F., Semel, M., Carter, B. D., Rees, D. E., \& Collier Cameron, A. 1997, MNRAS, 291, 658

Fabas, N. 2008, Master Thesis, Lulea University of Technology

Fossey, S. J. 1991, Nature, 353, 393

Furton, D. G., \& Witt, A. N. 1990, ApJ, 364, L45

Geballe, T. R., Tielens, A. G. G. M., Allamandola, L. J., Moorhouse, A., \& Brand, P. W. J. L. 1989, ApJ, 341, 278

Gledhill, T. M., Witt, A. N., Vijh, U. P., \& Davis, C. J. 2009, MNRAS, 392, 1217

Glinski, R. J., \& Anderson, C. M. 2002, MNRAS, 332, L17

Glinski, R. J., Michaels, P. D., Anderson, C. M., et al. 2009, Ap\&SS, 323, 337

Gordon, K. D., Witt, A. N., \& Friedmann, B. C. 1998, ApJ, 498, 522

Greenstein, J. L., \& Oke, J. B. 1977, PASP, 89, 131

Hony, S., Van Kerckhoven, C., Peeters, E., et al. 2001, A\&A, 370, 1030

Merrill, K. M. 1977, in The Interaction of Variable Stars with their Environment, ed. R. Kippenhahn, J. Rahe, \& W. Strohmeier, IAU Coll., 42, 446

Nathanson, G. M., \& McClelland, G. M. 1986, J. Chem. Phys., 85, 4311

Peeters, E., Hony, S., Van Kerckhoven, C., et al. 2002, A\&A, 390, 1089

Perkins, H. G., Scarrott, S. M., Murdin, P., \& Bingham, R. G. 1981, MNRAS, 196,635

Reese, M. D., \& Sitko, M. L. 1996, ApJ, 467, L105

Russell, R. W., Soifer, B. T., \& Merrill, K. M. 1977, ApJ, 213, 66

Russell, R. W., Soifer, B. T., \& Willner, S. P. 1978, ApJ, 220, 568

Sarre, P. J. 1991, Nature, 351, 356

Sarre, P. J. 2006, J. Mol. Spec., 238, 1

Sarre, P. J., Miles, J. R., \& Scarrott, S. M. 1995, Science, 269, 674

Scarrott, S. M., Watkin, S., Miles, J. R., \& Sarre, P. J. 1992, MNRAS, 255, 11P

Schmidt, G. D., \& Witt, A. N. 1991, ApJ, 383, 698

Schmidt, G. D., Cohen, M., \& Margon, B. 1980, ApJ, 239, L133

Sharp, R. G., Reilly, N. J., Kable, S. H., \& Schmidt, T. W. 2006, ApJ, 639, 194

Sironi, L., \& Draine, B. T. 2009, ApJ, 698, 1292

Sloan, G. C., Grasdalen, G. L., \& Levan, P. D. 1993, ApJ, 409, 412

Song, I., McCombie, J., Kerr, T. H., \& Sarre, P. J. 2007, MNRAS, 380, 979

Tielens, A. G. G. M. 2008, ARA\&A, 46, 289

Tolkachev, V. A. 1998a, J. Appl. Spec., 65, 883

Tolkachev, V. A. 1998b, J. Appl. Spec., 65, 643

Tolkachev, V. A. 2002, J. Appl. Spec., 69, 711

Tolkachev, V. A., \& Blokhin, A. P. 2009, J. Appl. Spec., 76, 806

Tolkachev, V. A., \& Pliska, S. P. 1986, Opt. Spec., 61, 48

Tolkachev, V. A., \& Polubisok, S. A. 1995, Spec. Lett., 28, 441

Tolkachev, V. A., \& Polubisok, S. A. 1998, Opt. Spec., 84, 839

Van Winckel, H., Cohen, M., \& Gull, T. R. 2002, A\&A, 390, 147

Vijh, U. P., Witt, A. N., \& Gordon, K. D. 2004, ApJ, 606, L65

Vijh, U. P., Witt, A. N., \& Gordon, K. D. 2005, ApJ, 633, 262

Wada, S., Mizutani, Y., Narisawa, T., \& Tokunaga, A. T. 2009, ApJ, 690, 111

Waelkens, C., Van Winckel, H., Trams, N. R., \& Waters, L. B. F. M. 1992, A\&A, 256, L15

Waelkens, C., Waters, L. B. F. M., de Graauw, M. S., et al. 1996, A\&A, 315, L245

Warren-Smith, R. F., Scarrott, S. M., \& Murdin, P. 1981, Nature, 292, 317

Waters, L. B. F. M., Waelkens, C., Van Winckel, H., et al. 1998, Nature, 391, 868

Witt, A. N., \& Boroson, T. A. 1990, ApJ, 355, 182

Witt, A. N., Gordon, K. D., Vijh, U. P., et al. 2006, ApJ, 636, 303 